Check for updates

Cite this: Mater. Horiz., 2019, 6,412

DOI: $10.1039 / c 9 m h 90005 e$

rsc.li/materials-horizons

\section{Correction: Focused ion beam milling of self-assembled magnetic superstructures: an approach to fabricate nanoporous materials with tunable porosity}

\author{
Verner Håkonsen, ${ }^{a}$ Gurvinder Singh, ${ }^{\text {bc }}$ Jianying $\mathrm{He}^{\mathrm{a}}$ and Zhiliang Zhang ${ }^{\star a}$
}

Correction for 'Focused ion beam milling of self-assembled magnetic superstructures: an approach to fabricate nanoporous materials with tunable porosity' by Verner Håkonsen et al., Mater. Horiz., 2018, 5, 1211-1218.

The axis labels of Fig. $3 \mathrm{f}$ and $4 \mathrm{f}$ were not displayed in the originally published version of the manuscript. The correct versions of Fig. 3 and 4 are shown below.

The authors would also like to correct a mistake in the Introduction. On page 1211, in the first paragraph of the Introduction the sentence "Here, we fabricate self-assembled superstructures in different morphologies from magnetic nanoparticles of different shapes, and investigate their interaction with a Ga+-ion beam by means of the focus ion beam (FIB) milling technique." should read "Here, we fabricate self-assembled superstructures in different morphologies from magnetic nanoparticles of different shapes, and investigate their interaction with a $\mathrm{Ga}^{+}$-ion beam by means of the focused ion beam (FIB) milling technique."

\footnotetext{
${ }^{a}$ NTNU Nanomechanical Lab, Department of Structural Engineering, Norwegian University of Science and Technology (NTNU), Trondheim 7491, Norway.

E-mail: zhiliang.zhang@ntnu.no

${ }^{b}$ Department of Materials Science and Engineering, Norwegian University of Science and Technology (NTNU), Trondheim 7491, Norway

${ }^{c}$ School of Aerospace, Mechanical and Mechatronic Engineering, University of Sydney, Sydney, NSW 2008, Australia
} 

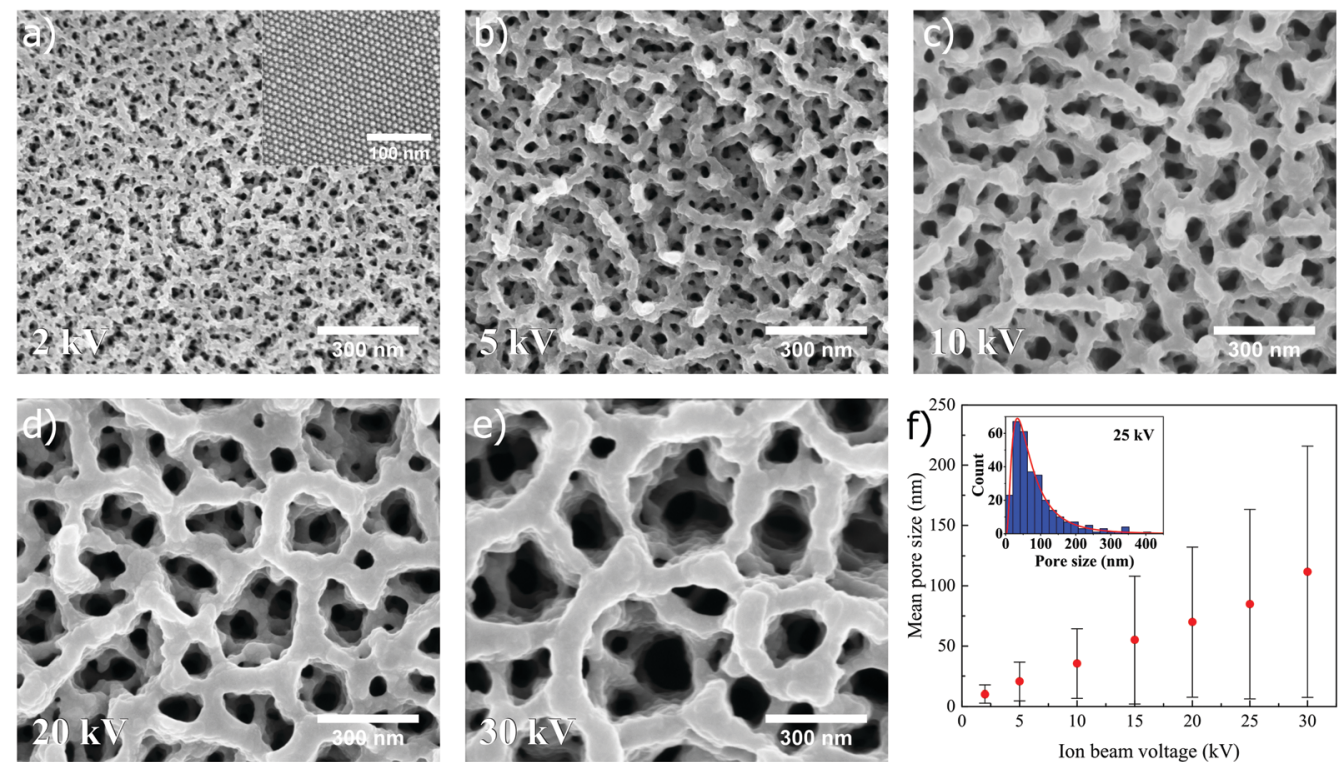

Fig. 3 Tuning the porosity of the resulting pore network during ion beam exposure of superstructures composed of nanospheres, by controlling the ion beam voltage at a constant dose of $0.5 \mathrm{nC} \mu \mathrm{m}^{-2}$. (a-e) Show SEM micrographs of the resulting pore network after ion beam exposure at 2, 5, 10, 20 and $30 \mathrm{kV}$, respectively. (f) The measured pore sizes were fitted to a lognormal distribution, and mean values were found to increase in a linear manner with increasing ion beam voltage.
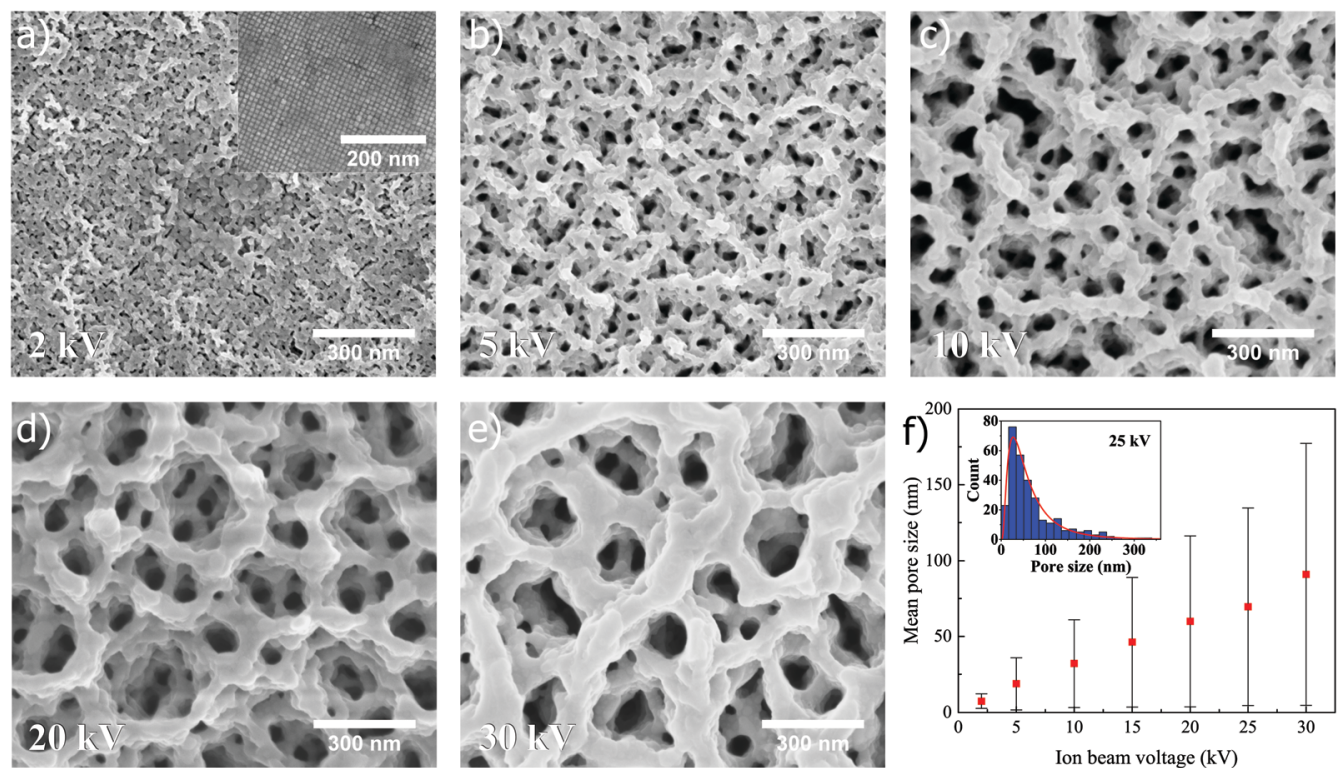

Fig. 4 Tuning the porosity of the resulting pore network during ion beam exposure of superstructures composed of nanocubes, by controlling the ion beam voltage at a constant dose of $0.5 \mathrm{nC} \mu \mathrm{m}^{-2}$. (a-e) Show SEM micrographs of the resulting pore network after ion beam exposure at 2, $5,10,20$ and $30 \mathrm{kV}$, respectively. (f) The measured pore sizes were fitted to a lognormal distribution, and mean values were found to increase in a linear manner with increasing ion beam voltage.

The Royal Society of Chemistry apologises for these errors and any consequent inconvenience to authors and readers. 